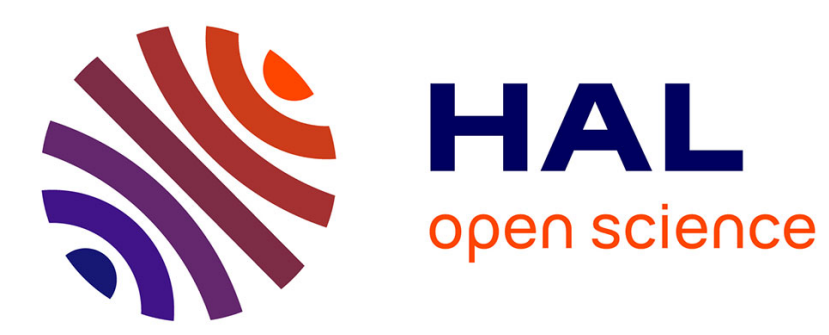

\title{
Dynamic adaptive management of buffer for QoS guarantees and energy reduction
}

Wael Zouaoui, Carolina Albea-Sanchez, Yann Labit

\section{To cite this version:}

Wael Zouaoui, Carolina Albea-Sanchez, Yann Labit. Dynamic adaptive management of buffer for QoS guarantees and energy reduction. Computer Software and Applications Conference Workshops (COMPSACW), 2014 IEEE 38th International, Jul 2014, Västerås, Sweden. pp.602 - 607, 10.1109/COMPSACW.2014.101 . hal-01068657

\section{HAL Id: hal-01068657 https://hal.science/hal-01068657}

Submitted on 26 Sep 2014

HAL is a multi-disciplinary open access archive for the deposit and dissemination of scientific research documents, whether they are published or not. The documents may come from teaching and research institutions in France or abroad, or from public or private research centers.
L'archive ouverte pluridisciplinaire HAL, est destinée au dépôt et à la diffusion de documents scientifiques de niveau recherche, publiés ou non, émanant des établissements d'enseignement et de recherche français ou étrangers, des laboratoires publics ou privés. 


\section{Dynamic adaptive management of buffer for QoS guarantees and energy reduction}

\author{
Wael Zouaoui \\ CNRS, LAAS, \\ 7, avenue du Colonel Roche \\ F- 31400 Toulouse France \\ Univ de Toulouse, UPS, LAAS, \\ F- 31400 Toulouse France \\ wzouaoui@laas.fr
}

\author{
Carolina Albea Sanchez \\ CNRS, LAAS, \\ 7, avenue du Colonel Roche \\ F- 31400 Toulouse France \\ Univ de Toulouse, UPS, LAAS, \\ F- 31400 Toulouse France \\ calbea@laas.fr
}

\author{
Yann Labit \\ CNRS, LAAS, \\ 7, avenue du Colonel Roche \\ F- 31400 Toulouse France \\ Univ de Toulouse, UPS, LAAS, \\ F- 31400 Toulouse France \\ ylabit@laas.fr
}

\begin{abstract}
Technological advances in communication networks have made to increase communication rate as well as energy consumption. Some equipments and protocol solutions have been developed to treat these problems e.g. Smart Sleeping and Rate Adaptation. We present an original and alternative adaptive solution from Automatic community to control the output flow of a node, in such a way that Quality of Service (QoS) improves and energy consumption is reduced. The controller is developed by using optimal control theory while guaranteeing asymptotic stability (Lyapunov stable) and avoiding losses. In order to validate this approach, we apply the adaptive controller in "Matlab-Simulink" and in the network simulator "NS-2". Within ADREAM framework ${ }^{1}$, this "control green" will allow to improve energy consumption regarding traffic load and available energy in communication networks.
\end{abstract}

Keywords-Green Network, Adaptive system, Quality of Service, Energy, ADREAM, Control theory.

\section{INTRODUCTION}

Since few years, the number of communication system users has significantly gone up, thereby energy consumption have also increased. According to [1], the number of Internet users reached 2.4 billion of the population in 2012. Consequently, the energy consumption in communication networks represents $5.5 \%$ of annual world electricity production and a rate of $2 \%$ of carbon dioxide emissions $\left(\mathrm{CO}_{2}\right)$. The perspective for 2015 , with probably 2.8 billion of Internet users, is to achieve a traffic 4 times larger and a double energy consumption (approximately 25\% larger per year) [2]. This issue requires to research new ways to reduce energy consumption.

Some works have already been developed (mainly in the context of wireless networks) and several hardware and protocol solutions have been proposed to reduce energy consumption in communication networks included the following ones:

- ALR (Adaptive Link Rate): this idea adapts transmission rates depending and energy threshold in the nodes [3].

- Smart sleeping: this proposition consists in switching off the inactive or idle routers[4], [5].

\footnotetext{
${ }^{1}$ http://www.laas.fr/ADREAM/
}

- Resizing network: this solution is complex and expensive, it modifies the architecture of communication networks [6].

- Energy-aware routing: it calculates the best route in order to minimize the number of routers within the Quality of Service (QoS), particularly in terms of energy consumption [7].

In the industry domain, some important companies in the sector have already started a "green revolution" (development of "green" network equipments)). Among these companies, we cite the following ones: Cisco (EnergyWise), Alcatel-Lucent (GreenTouch), IBM (Big-Green), NetGear (routers, energyware switches) Intel, Microsoft, Toshiba, Hewlett-Packard (Advanced Configuration and Power Interface).

In this paper, a control mechanism is proposed from control theory. The control is locally implemented at each selected node of a network topology. The choice of the selected nodes is a problem in itself, and it is not an issue in this work. The adaptive law controls the traffic that crosses a node, by using the length-queue where packets are temporarily stored. This proposed law guarantees unsaturated output traffic to avoid packet loss, which ensures, simultaneously, a fast convergence to a reference level. This reference level in the buffer is given by the energy thresholds proposed by the ALR model, which ensures energy reduction [8].

The proposed controller is validated in Matlab-Simulink [9] and NS-2 [10]. Likewise, energy consumption measures are given by ECOFEN [8] (implemented module in NS-2).

The rest of this paper is organized as follows: Section I presents the ALR model and the ECOFEN module. In Section II, a mathematical model and a controller are proposed. Finally, Section II presents the results of simulations in MatlabSimulink and NS-2.

\section{ALR MODEL AND ECOFEN MODULE}

Firstly, let model the energy consumption of the network equipment. For this purpose, we consider a router model designed in NS-2. NS-2 is one of the most currently simulators used in network community [10]. This simulator allows us to simulate large networks with realistic traffic and to obtain QoS measures. 
The ECOFEN module (Energy Consumption Model for End-to-end Networks) was developed in NS-2. This module provides the instantaneous energy consumption of each equipment taking into account the traffic and the type of employed equipment (router, switch, network card, etc.) [8]. This module is based on the ALR model (Adaptive Link Rate) which adapts transmission rates depending on link use-rate.

The power consumption mathematical equations are

$$
P= \begin{cases}P_{0} & \text { si } w_{n}=0 \\ a_{1} w_{n}+P_{1} & \text { si } \left.\left.w_{n} \in\right] 0 ; W_{1}\right] \\ \vdots & \\ a_{i} w_{n}+\left(P_{i}-a_{i} W_{i-1}\right) & \text { si } \left.\left.w_{n} \in\right] W_{i-1} ; W_{i}\right] \\ \vdots & \\ a_{N} w_{n}+\left(P_{N}-a_{n} W_{N-1}\right) & \text { si } \left.\left.w_{n} \in\right] W_{N-1} ; W_{N}\right]\end{cases}
$$

with:

- $\quad a_{i} \in \mathbb{R}^{+}$: the slope of the different linear section.

- $\quad w_{n} \in \mathbb{R}$ : the input traffic of each network element.

- $\quad W_{i} \in \mathbb{R}^{+}:$the use-rate thresholds.

- $\quad P_{i} \in \mathbb{R}^{+}$: the start power of each different stage.

Figure 1 shows the power model for a router.

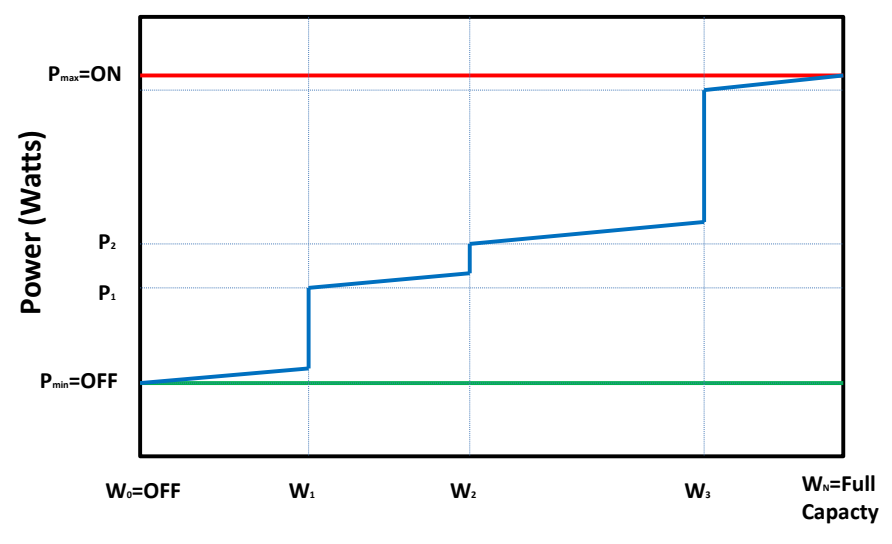

Bandwidth (MB/s)

Fig. 1. ALR model of power consumption in a network element (router model).

In this model, the input traffic depends on the consumed energy by the network element.

\section{A. Router energy model}

Let take previous model for studying energy consumption in a router. For this issue, we control input traffic, $w_{n}$, of the controlled node. We artificially and temporarily delay a certain number of packets in a buffer to a pre-defined value $q_{\text {ref }}$. The length-queue reference $q_{r e f}$ is given according to the input traffic in the router. This selection may provide some problems in network QoS, in terms of "delay". In other words, there is a energy consumption reduction and transmission delay trade-off.

Therefore, model (1) is rewritten

$$
P= \begin{cases}P_{0} & \text { si } w_{n}=0 \\ a_{1} w_{n}+P_{1}+b q_{r e f} & \text { si } \left.\left.w_{n} \in\right] 0 ; W_{1}\right] \\ \vdots & \\ a_{i} w_{n}+\left(P_{i}-a_{i} W_{i-1}\right)+b q_{r e f} & \text { si } \left.\left.w_{n} \in\right] W_{i-1} ; W_{i}\right] \\ \vdots & \\ a_{N} w_{n}+\left(P_{n}-a_{N} W_{N-1}\right)+b q_{r e f} & \text { si } \left.\left.w_{n} \in\right] W_{N-1} ; W_{N}\right]\end{cases}
$$

with :

- $q_{\text {ref }}$ : the queue length reference.

- $\quad b$ : a coefficient of the queue length reference $q_{r e f}$.

A reliable adaptation of the queue length reference is mandatory in order to reduce energy consumption with QoS guarantees.

This selection of $q_{r e f}$ depends on two key parameters:

- the update time-window for the queue length reference.

$$
T_{q_{\text {ref }}}=\frac{T_{w}}{\beta},
$$

where $T_{w}$ is the time-window related to the node input traffic. $\beta$ is the number of changes of the value of $q_{\text {ref }}$ during the period $T_{w}$. This allows to update more or less frequently $q_{\text {ref }}$ depending on the traffic variability.

- The queue length reference

$$
q_{\text {ref }}=\frac{\hat{w}_{n}}{\gamma},
$$

where $\hat{w}_{n}$ is the average input traffic on a fixed timewindow $T_{w} \cdot \gamma$ is a step (weighting coefficient) for adapting the amplitude of the reference function $w_{n}$.

Remark. The judicious choice of the input traffic average $\hat{w}_{n}$, on a fixed time window $T_{w}$, take into account the high variability of this traffic.

\section{TRAFFIC CONTROL}

\section{A. Network model}

One of the most important challenge is to find a suitable network model for control purposes. In this section, an average simple network model is developed inspired by a flow system. This reasonable simple model can handle a vast of networks behaviors (see [11], [12]). Thus, the model is proposed to be

$$
q_{k+1}=q_{k}-T_{s}\left(u_{k}-\hat{w}_{n}\right)
$$

with:

- $\quad q_{k} \in \mathbb{R}^{+}$: the average queue length node and it is a state variable.

- $T_{s} \in \mathbb{R}^{+}$: the sampling time of the network model.

- $u_{k} \in \mathbb{R}$ : the output traffic of each node. This is the control variable. 
- $\hat{w}_{n}$ : the mean input traffic of each node and it is considered constant.

Note that this is a discrete-time model.

\section{B. Control law}

\section{a) Error equation for an selected node: :} is:

Taking as error variable $e_{k} \triangleq q_{r e f}-q_{k}$, the error equation

$$
e_{k+1}=e_{k}+T_{s}\left(u_{k}-\hat{w}_{n}\right) \text {. }
$$

The proposed control law is:

$$
u_{k}=\operatorname{sat}_{0}^{C}\left\{u_{k-1}+K x_{k}\right\},
$$

where $K=\left[\begin{array}{ll}K_{1} & K_{2}\end{array}\right]$ are the control gains and $x_{k}=$ $\left[\begin{array}{ll}e_{k} & e_{k-1}\end{array}\right]^{T}$. The high-bound saturation $C$ depends on the network capacity.

Remark. The saturation function is added in order to satisfy the physical constraint of the network links, whose capacity has to be positive and less than $C\left(\right.$ or $W_{N}$ in Fig. 1).

b) Polytopic representation for the saturated control (7) and the error equation (6): :

The control law (7) presents an isolated nonlinearity which may be difficult to consider if we aim at designing effectively a controller. Therefore, the saturation is modeled as a polytopic model, which will be used to get efficient design methods as presented in the following lemmas. Define $\chi_{k} \triangleq\left[\begin{array}{ll}u_{k-1} & x_{k}^{T}\end{array}\right]^{T}$.

The following lemma recalls how to encapsulate the saturation function into a polytope.

Lemma 1. [13]. Given $K, G \in \mathbb{R}^{1 \times 2}$, for all, $\chi_{k} \in \mathbb{R}^{3 \times 1}$, if $\chi_{k} \in\left\{\chi_{k} \in \mathbb{R}^{1 \times 3}:\left[\begin{array}{ll}1 & G\end{array}\right] \chi_{k} \in\left[\begin{array}{ll}0 & C\end{array}\right]\right\}$, then

$$
\operatorname{sat}_{0}^{C}\left\{\left[\begin{array}{ll}
1 & G
\end{array}\right] \chi_{k}\right\} \in \mathcal{C} \mathrm{O}\left\{\left[\begin{array}{ll}
1 & K
\end{array}\right] \chi_{k}, \quad\left[\begin{array}{ll}
1 & G
\end{array}\right] \chi_{k}\right\} .
$$

The next lemma proposes then a polytopic representation for the PI (Proportional Integral) control law :

Lemma 2. Assume there exist $G \in \mathbb{R}^{1 \times 2}, c>0$ and $\Psi \triangleq$ $\left[\begin{array}{cc}\Theta_{1} & \Theta_{2} \\ \Theta_{2}^{T} & P\end{array}\right]>0 \in \mathbb{R}^{3}$ where $P \in \mathbb{R}^{2}$ is a positive definite matrix such that for any $\chi_{k} \in \mathfrak{X}$, where

$$
\mathfrak{X}=\left\{\chi_{k}: \chi_{k}^{T} \Psi \chi_{k} \leq c^{-1}\right\},
$$

then, $0<u_{k-1}+G x_{k}<C$, and control law (7) admits the following representation

$$
\begin{aligned}
u_{k} & =\alpha_{k}\left(u_{k-1}+K x_{k}\right)+\left(1-\alpha_{k}\right)\left(u_{k-1}+G x_{k}\right) \\
& =u_{k-1}+\left(\alpha_{k} K x_{k}+\left(1-\alpha_{k}\right) G x_{k}\right)=u_{k-1}+\bar{u}_{k},
\end{aligned}
$$

where $\bar{u}_{k} \triangleq\left(\alpha_{k} K+\left(1-\alpha_{k}\right) G\right) x_{k}$ with $\alpha_{k} \in[0,1]$, for all $k>0$.

Then, Eq. (6) can be rewritten as:

$$
e_{k+1}=e_{k}+T_{s}\left(u_{k-1}+\bar{u}_{k}\right)-T_{s} \hat{w}_{n}
$$

From Lemma 1 and 2 and from [14], we obtain:

$$
x_{k+1}=A x_{k}+B \bar{u}_{k},
$$

where

$$
A \triangleq\left[\begin{array}{cc}
2 & -1 \\
1 & 0
\end{array}\right], B \triangleq\left[\begin{array}{c}
T_{s} \\
0
\end{array}\right]
$$

Equation (10) can be rewritten in the following explicit closedloop form:

$$
x_{k+1}=A x_{k}+B\left(\alpha_{k} K+\left(1-\alpha_{k}\right) G\right) x_{k} .
$$

c) Condition for unsaturated control: To ensure that the control signal do not saturate, we need to enforce that:

$$
0<u_{k-1}+G x_{k}<C, \quad \forall \chi_{k} \in \mathfrak{X}
$$

where $\mathfrak{X}$ is given in (8).

Subtracting $\frac{C}{2}$ in inequality (12) and from [15], it is seen that, (12) is satisfied if:

$$
\begin{aligned}
C & >c x_{k}^{T} P x_{k}+c u_{k-1} \Theta_{1} u_{k-1}+c x_{k}^{T} \Theta_{2}^{T} u_{k-1} \\
& +c u_{k-1} \Theta_{2} x_{k}-1+C>2 u_{k-1}+2 G x_{k}-C .
\end{aligned}
$$

From Lemma 2, nequalities (13) can be rewritten as:

$$
\left[\begin{array}{c}
1 \\
\pm u_{k-1} \\
\pm x_{k}
\end{array}\right]^{T}\left[\begin{array}{ccc}
2 C-1 & -1 & -G \\
-1 & c \Theta_{1} & c \Theta_{2} \\
-G^{T} & c \Theta_{2}^{T} & c P
\end{array}\right]\left[\begin{array}{c}
1 \\
\pm u_{k-1} \\
\pm x_{k}
\end{array}\right]>0
$$

Employing the Schur's complement and some mathematical manipulations, defining $Y_{i} \triangleq G_{i} Q_{i}$ with $Q_{i} \in \mathbb{R}^{2}$ an Hermitian matrix, applying $Q_{i}=P_{i}^{-1}$ and pre- and postmultiplying by $\operatorname{diag}\left\{I_{2}, I_{2}, Q_{i}\right\}$, it can be shown that this last inequality is satisfied if

$$
\Lambda \triangleq\left[\begin{array}{ccc}
c & -1 & -Y \\
-1 & (2 C-1) \Theta_{1} & (2 C-1) \Theta_{2} Q \\
-Y^{T} & (2 C-1) Q \Theta_{2}^{T} & (2 C-1) Q
\end{array}\right]>0
$$

\section{Sub-Optimal robust saturated control design}

Firstly, we aim at minimizing the control signal $u_{k}$ as well as $x_{k}$, (i.e. $q_{k}$ converges to $q_{r e f}$, as fast as possible). For this purpose, we propose to minimize a quadratic cost function:

$$
J=\sum_{k=0}^{\infty}\left(x_{k}^{T} \mathcal{Q} x_{k}+u_{k} \mathcal{R} u_{k}\right)
$$

where $\mathcal{Q}>0$ and $\mathcal{R}>0$, which implies that $J$ is strictly positive.

Secondly, as the sampling period $T_{s}$ of the network is supposed to be constant, we aim also at designing this suboptimal gain $K$ for the control law (7) in such a way that the system (5) is robust with respect to parameter uncertainty. 
Lemma 3. Assume there exist $\mathcal{Q} \in \mathbb{R}^{2}, \mathcal{R} \in \mathbb{R}$ and $P>0$ associated with a Lyapunov function candidate $V_{k}$, such that,

$$
V_{k+1}-V_{k}+x_{k}^{T} \mathcal{Q} x_{k}+u_{k} \mathcal{R} u_{k}<0,
$$

then the performance index $J$ is minimized at the same time that system stability is guaranteed.

Proof: By summing the inequality (17) from $k=0$ to $k=\infty$, we obtain:

$$
\begin{aligned}
& \sum_{k=0}^{\infty}\left(V_{k+1}-V_{k}+x_{k}^{T} \mathcal{Q} x_{k}+u_{k} \mathcal{R} u_{k}\right) \\
& =V_{\infty}-V_{0}+J<-V_{0}+J<0,
\end{aligned}
$$

thus $J<V_{0} \leq x_{0}^{T} P x_{0}$, then the quadratic cost function $J$ is minimized, and the system stability is ensured.

The uncertain parameter $\alpha_{k}$ given above is now taken into account. We chose the extremes of this parameter $\left(\alpha_{k}^{(j)}=0,1\right.$ for $j=\{1,2\}$ ) as the vertices of the polytope.

Next, a sufficient condition to satisfy lemma 3 is derived.

Theorem 1. Consider system (11) and control law $u_{k}=$ $u_{k-1}+\alpha_{k}^{(j)} K x_{k}+\left(1-\alpha_{k}^{(j)}\right) G x_{k}$ for $\alpha_{k}^{(j)} \in[0,1]$, where $K, G, S^{(j)} \in \mathbb{R}^{1 \times 2}$. If there exist $T, Y \in \mathbb{R}^{2 \times 1}$ and $Q=$ $P^{-1}, \mathcal{Q} \in \mathbb{R}^{2}$ with $K=T Q^{-1}, G=Y Q^{-1}$ and $\mathcal{R}, c, \mu>0$ for $j=1,2$ such that

$$
\begin{array}{rl}
\min _{K} & c, \mu \\
& P>0 \\
& \Gamma_{k}^{(j)}<0 \quad j=1,2, \\
& -\mu I_{2}+Q^{-1}<0 \\
& \Lambda>0
\end{array}
$$

being $\Gamma_{k}^{(j)}$ defined in (22) (given at the top of next page), are satisfied. Then, for all initial conditions in $\mathfrak{X}$, the states converge asymptotically to the equilibrium. The closed-loop system (11) is unsaturated and sub-optimum with respect to $J$.

Proof: The goal is to satisfy $\Gamma_{k}^{(j)}<0$ for both asymptotic stability and minimization of $J$ in the entire polytope $\Upsilon_{j}$.

Then,

$$
\begin{aligned}
& V_{k+1}^{(j)}-V_{k}^{(j)}+x_{k}^{T} \mathcal{Q} x_{k}+u_{k} \mathcal{R} u_{k}=x_{k+1}^{T} P x_{k+1} \\
-\quad & x_{k}^{T} P x_{k}+x_{k}^{T} \mathcal{Q} x_{k}+\left(u_{k-1}+K x_{k}\right) \mathcal{R}\left(u_{k-1}+K x_{k}\right) \\
= & x_{k}^{T}\left[\bar{A}^{T} P \bar{A}-P+\mathcal{Q}+\left(S^{(j)}+K\right) \mathcal{R}\left(S^{(j)}+K\right)\right] x_{k}
\end{aligned}
$$

where $\bar{A}^{(j)} \triangleq A+\alpha_{k}^{(j)} K^{T}+\left(1-\alpha_{k}^{(j)}\right) G^{T}$ for $j=1,2$ and $S^{(j)} \triangleq\left[T_{s}^{-1}-T_{s}^{-1}\right]$. Straightforwardly, applying Schur's complement and pre- and post-multiplying by $\left\{Q_{1}, I_{2}, I_{2}, I_{2}\right\}$, it can be shown that if $\Gamma_{k}^{(j)}<0$ then conditions for using Lemma 3 are satisfied and the closed loop stability is guaranteed. Furthermore, minimizing $\mu>0$, such that, $P<\mu I_{2}$, allow to minimize $J$.

The sub-optimal gain $K$, obtained applying Theorem 1 guarantees both stability model of communication model, a transitional regime control and a controlled QoS.

\section{Analytic \& Simulation Results}

The control gains for the node, control (7) is computed. To validate this approach, we take a simple topology composed of two nodes and one source, as shown in Figure 2.

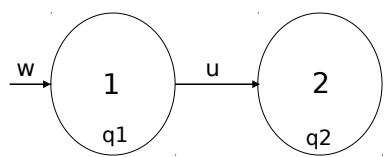

Fig. 2. Network node for validation

We choose: $C=25000 \mathrm{pqts} / \mathrm{s}, T_{\mathrm{s}}=0.05 \mathrm{~s}$. From Theorem 1, it comes that: $K_{1}=-21$ and $K_{2}=20$. Likewise $c=$ $0.078, \mu=0.12, \Theta_{1_{i}}=0.0003, \Theta_{2_{i}}=\left[\begin{array}{ll}-0.005 & 0.004\end{array}\right] G=$ $\left[\begin{array}{ll}-14.3 & 13.1\end{array}\right], \mathcal{R}=0.001, \mathcal{Q}=\left[\begin{array}{cc}0.001 & 0 \\ 0 & 0.0001\end{array}\right]$, $P=\left[\begin{array}{cc}0.08 & -0.05 \\ -0.05 & 0.06\end{array}\right], \gamma=1000, \beta=1$

Two types of simulations, using $T_{s}=0.05 \mathrm{~s}$, are performed. Firstly, we test the control law under Matlab-Simulink $(A)$ to validate the assumption to take an average-model for computing a control law. Then, simulations are performed in NS-2 $(B)$. These two simulators have been choosing for the following raison: NS-2 runs with events and Matlab-Simulink with discret time. Note that the modified ECOFEN model is implemented under NS-2.

In Figures 3 and 5, the evolution of the input traffic generated by the source and the output traffic calculated from the control law are presented. Likewise, it is shown in Figures 4 and 6, the queue length evolution. Note, that the MatlabSimulink simulations are closed to NS-2 simulations. Thus, the simplification made about the average-model is validated. In addition, network traffic is unsaturated, so losses are avoided.

Figure 7 shows the power consumption with and without control law. Note, there is a significant improvement in power consumption from $0 s$ to $6.2 s$ which represents $62 \%$ of the total simulation time $(10 s)$. During this time period, packets are stored in the queue (with size $q_{r e f}$ ), the output traffic is smaller than the input traffic. Moreover, power is smaller than a power threshold, hence the reduction of the energy consumption. Given the ALR model, if the output traffic is smaller than a threshold $W_{i}$ (see 1), the consumed energy will be smaller than without control. On the other hand, from $6.2 \mathrm{~s}$ to $10 s$, queue length releases packets already stored. Hence, the output traffic is larger than the input traffic, what leads to a slight energy overconsumption, the difference is less than the reduction.

Note that the minimum power consumption of the ALR model is $P_{0}=155 \mathrm{~W}$ (Figure 7). This value is obtained when there is not traffic crossing the controlled node $\left(w_{n}=\right.$ $0 \mathrm{Mb} / \mathrm{s}$ ). This is a minimum power consumption of the operational node without traffic.

In Figure 8, the two phases of energy consumption (reduced and increased energy consumption) are easily shown. In this simulation, it is shown that the global energy consumption for 


$$
\Gamma_{k}^{(j)} \triangleq\left[\begin{array}{cccc}
-Q & Q \bar{A}^{(j)^{T}}+\left(\alpha_{k}^{(j)} T^{T}+\left(1-\alpha_{k}^{(j)}\right) Y^{T}\right) B^{(j)} & -Q & -\bar{A}^{(j)^{T}}+\alpha_{k}^{(j)} T^{T}+\left(1-\alpha_{k}^{(j)}\right) Y^{T} \\
* & -Q & 0 & 0 \\
* & * & -\mathcal{Q}^{-1} & 0 \\
* & * & * & -(1+\mathcal{R})^{-1}
\end{array}\right]
$$

a time period of $10 \mathrm{~s}$ is reduced $12.2 \%$ (energy gain) in the ALR model. The ratio between reduced and increased energy consumption is up to $60 \%$.

\section{A. Simulation Matlab-Simulink}

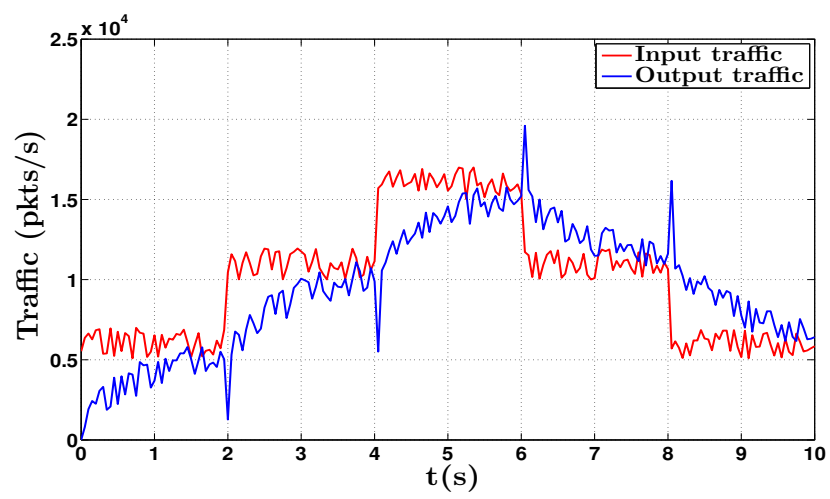

Fig. 3. Input (red) and output (blue) traffic.

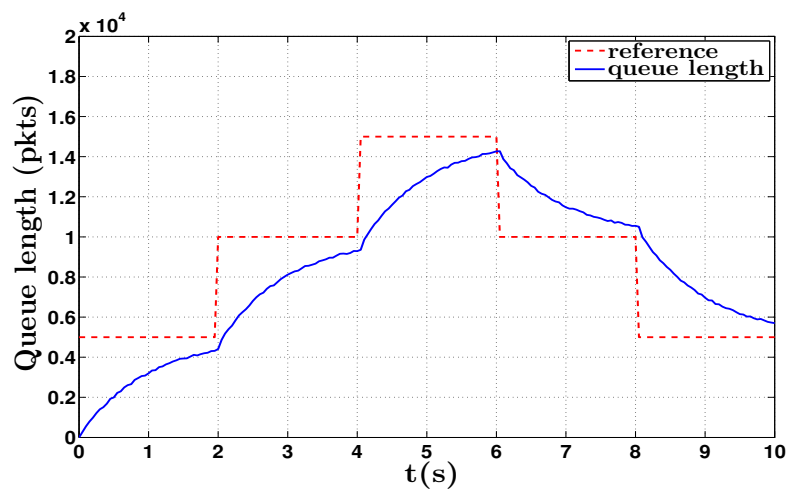

Fig. 4. Queue length evolution (blue) and queue length reference (red).

\section{B. Simulation NS2}

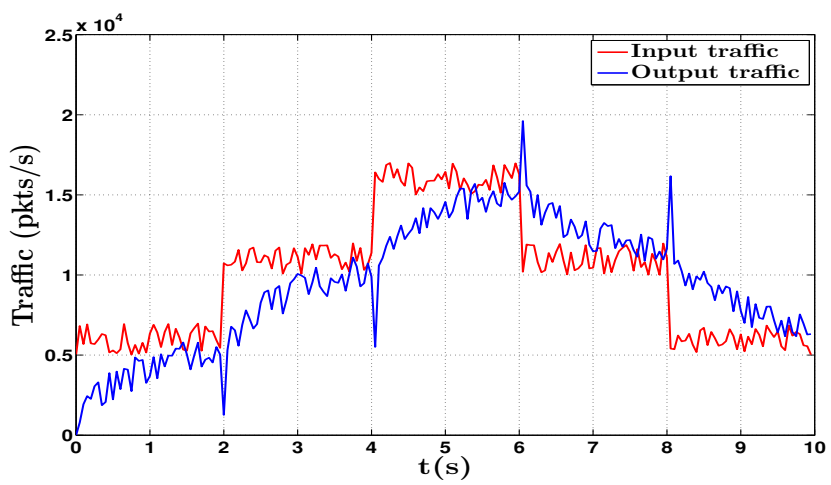

Fig. 5. Input (red) and output (blue) traffic

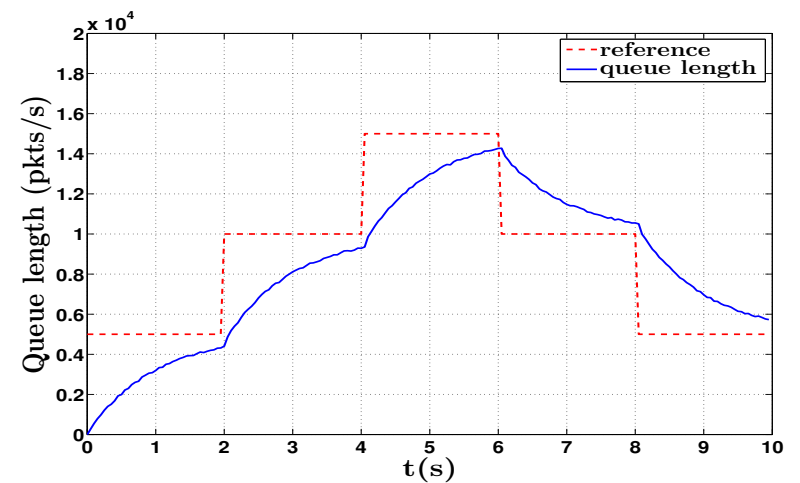

Fig. 6. Queue length evolution (blue) and queue length reference (red).

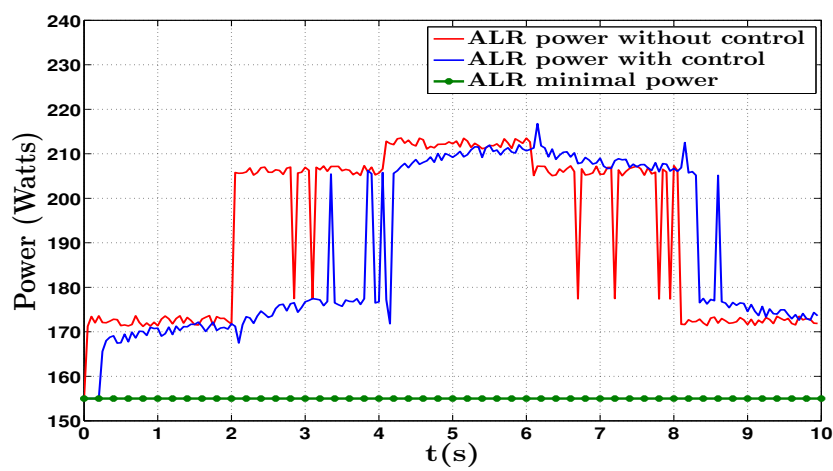

Fig. 7. Power consumption with (blue) and without (red) flow control, on ALR model of a network node.

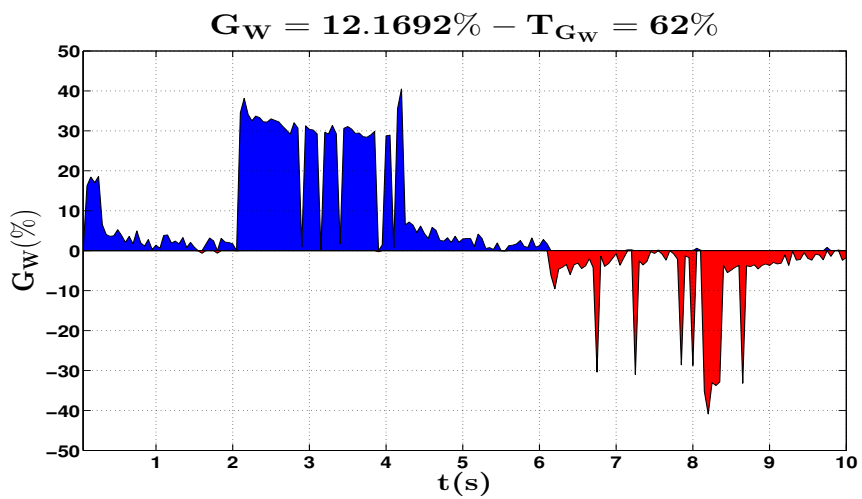

Fig. 8. Power gain (in blue).

\section{CONCLUSION}

In this article, an alternative adaptive solution for reducing energy consumption in networks has been presented. This solution is developed from control theory. The controller was 
developed taking into account both the limitation of linkcapacities and minimization of a quadratic costs to ensure the fast convergence of the queue-length. In such a way, that a level of QoS is guaranteed (under a trade-off). Simulations under Matlab-Simulink and NS-2 validate this control law. In addition, an energy gain is shown, what let to call our control "control green". Future work will improve the optimization phases for $(\gamma, \beta)$ parameters and the selection of adaptive nodes.

\section{REFERENCES}

[1] J. Koomey, "Worldwide electricity used in data centers," Environ. Res. Lett. 3 034008, 2008.

[2] Z. Zhang, A. S. Ackerman, G. Feingold, S. Platnick, R. Pincus, and H. Xue, "Effects of cloud horizontal inhomogeneity and drizzle on remote sensing of cloud droplet effective radius: Case studies based on large-eddy simulations," Journal of Geophysical Research: Atmospheres, vol. 117, no. D19, pp. n/a-n/a, 2012. [Online]. Available: http://dx.doi.org/10.1029/2012JD017655

[3] C. Gunaratne, K. Christensen, B. Nordman, and S. Suen, "Reducing the energy consumption of ethernet with adaptive link rate (alr)," Computers, IEEE Transactions on, vol. 57, no. 4, pp. 448-461, April 2008.

[4] A. Bianzino, C. Chaudet, D. Rossi, and J. Rougier, "A survey of green networking research," Communications Surveys Tutorials, IEEE, vol. 14, no. 1, pp. 3-20, First 2012.

[5] M. Jimeno, K. Christensen, and B. Nordman, "A network connection proxy to enable hosts to sleep and save energy," in Performance, Computing and Communications Conference, 2008. IPCCC 2008, p. 101110, 2008.

[6] L. Ceuppens, A. Sardella, and D. Kharitonov, "Power saving strategies and technologies in network equipment opportunities and challenges, risk and rewards," Applications and the Internet, 2008. SAINT 2008. International Symposium on, p. 381384, 2008.

[7] E. Amaldi, A. Capone, and L. Gianoli, "Energy-aware IP traffic engineering with shortest path routing," Computer Networks, p. 15031517, 2013.

[8] A. Orgerie, L. Lefèvre, and I. Guérin-Lassous, "Energy-efficient bandwidth reservation for bulk data transfers in dedicated wired networks," The Journal of Supercomputing, pp. 1-28, 2011.

[9] MATLAB, version 8.2.0.701 (R2013b). Natick, Massachusetts: The MathWorks Inc., 2013.

[10] K. Fall and K. Varadhan, "The ns manual," notes and documentation on the software ns2-simulator, 2002, uRL: www.isi.edu/nsnam/ns/.

[11] S. Mascolo, "Congestion control in high-speed communication networks using the smith principle," Automatica, vol. 35, no. 12, pp. 19211935, 1999.

[12] R. Srikant, The mathematics of Internet congestion control. Springer, 2004.

[13] T. Hu and Z. Lin, Control systems with actuator saturation: analysis and design. Birkhauser, 2001.

[14] C. Albea, F. Gordillo, and C. C. De Wit, "Robust saturated control for low-power circuits," Control Systems Technology, IEEE Transactions on, vol. 21, no. 2, pp. 530-537, 2013.

[15] E. Fridman, A. Pila, and U. Shaked, "Regional stabilization and $H_{\infty}$ control of time-delay systems with saturating actuators," International Journal of Robust and Nonlinear Control, vol. 13, no. 9, pp. 885-907, 2003. 